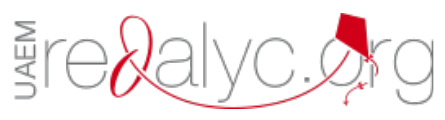

Centro Sur

ISSN: $2600-5743$

compasacademico@icloud.com

Grupo Compás

Ecuador

\title{
Gestión del talento humano basado en competencia en areas de sociales
}

López Vera, Franklin

Gestión del talento humano basado en competencia en areas de sociales

Centro Sur, vol. 2, núm. 1, 2018

Grupo Compás, Ecuador

Disponible en: http://www.redalyc.org/articulo.oa?id=588861692002 


\title{
Gestión del talento humano basado en competencia en areas de sociales
}

\author{
Management of human talent based on competition in social \\ areas \\ Franklin López Vera Franklin.lopezv@ug.edu.ec \\ Docente de la Universidad de Guayaquil, Ecuador \\ iD http://orcid.org/0000-0001-9923-3426
}

Centro Sur, vol. 2, núm. 1, 2018

Grupo Compás, Ecuador

Recepción: 16 Mayo 2017

Aprobación: 31 Mayo 2018

Redalyc: http://www.redalyc.org/ articulo.oa?id $=588861692002$
Resumen: Dentro del contexto organizacional, es el recurso humano uno de los aspectos de más alto impacto sobre el plan estratégico de la empresa, y su desarrollo en cuanto a cualificación y calificación, es uno de los puntos más tratados dentro del tema del aprendizaje organizacional, principalmente, lo relacionado con la formación en el trabajo. Sin embargo, cuando se habla del desarrollo de competencias laborales, se hace énfasis en aquellas específicas y propias de cada desempeño, las cuales se perfeccionan o mantienen a través de los antiguos modelos de capacitación o entrenamiento. Como objetivo general analizar los principales aspectos involucrados en la gestión del talento humano basada en competencias, las cuales mediante su desarrollo efectivo, se constituyen en fuente principal del logro de los objetivos empresariales acordes con las necesidades del entorno laboral y las exigencias del mercado.

Palabras clave: Aprendizaje Organizacional, Capital Humano, Competencias, Gestión de Talento Humano, Mercado.

Abstract: Within the organizational context, is the human resource aspect highest impact on the strategic plan of the company and its development in terms of qualification and qualification, it is one of the points most discussed under the topic of organizational learning, mainly, related to training at work. However, when speaking of skills development, and focus on those specific characteristics of each performance is made, which are improved or maintained through the old models or training. As a general objective to analyze the main aspects involved in the management of human talent based on competencies, which through its effective development, constitute the main source of the achievement of business objectives consistent with the needs of the working environment and market demands. To fulfill this objective, the following specific were raised: Identify organizational HR processes in which directly influences the focus of labor skills, show the relationship between job performance and competencies that determine it, the difference and importance of the development of basic, generic and specific human capital of the organization skills.

Keywords: Human Capital, Human Resource Management, Market, Organizational Learning, Skills.

\section{INTRODUCCIÓN}

El reconocimiento de la importancia de las personas dentro de las organizaciones ha sido motivo de mucho interés, con frecuencia se exigen resultados, eficiencia y eficacia sin considerar sus sentimientos, pensamientos y aportes, es por ello que debido al avance de la tecnología sobre los procesos de recursos humanos, la globalización y nuevas formas de gestionar las empresas, la gestión del talento humano se ha vuelto un reto en las empresas de hoy. 
En la actualidad lo que permite diferenciar una empresa de otra son las personas que la componen convirtiéndose en una ventaja competitiva ya que participan sus conocimientos, habilidades y competencias.

La gestión del talento humano ha evolucionado apareciendo nuevas técnicas como es el de Gestión por Competencias Laborales como un modelo integral de gestión que proporciona respuestas a las necesidades de las empresas.

La empresa de la actualidad ha evolucionado, los cambios que suceden en el mundo tienen una influencia notoria e importante en las decisiones que se toman y las acciones que se llevan a cabo en las organizaciones, es por esto que cada una de las piezas que las conforman debe de adaptarse de manera óptima a estos cambios y es aquí donde el recurso humano toma otra dimensión al considerarse que al aumentar sus capacidades y aptitudes podrá entregar el máximo esfuerzo a su trabajo y se sentirá orgulloso de su aportación a la empresa.

Es paradójico como en la era de la información la persona toma un valor muy importante para la estructura de una empresa, la gestión actual deberá estar sustentada en la gente, quienes necesitan entender el propósito de la organización para la cual se desempeña y comprender su misión en ella para lograr el éxito conjunto.

A medida que se acerca el nuevo milenio, las organizaciones se desarrollan cada vez más, con una clara tendencia hacia la unificación de principios y de formas de hacer negocios. Los procesos productivos, las acciones comerciales, la planificación financiera y otros factores son similares en las diferentes empresas, pertenezcan o no al mismo sector o al mismo país. En la actualidad, y por este motivo, es posible que una organización implante en otra un sistema de gestión que esté funcionando con éxito, realizando simplemente algunas modificaciones de adaptación.

Cada empresa desarrolla sus actividades en un entorno propio, pero las técnicas de gestión de los negocios han alcanzado un nivel de unificación global que no se había conocido antes. Por ello, es fundamental que el equipo directivo sea consciente de su ubicación para poder conocer y definir el entorno de la empresa dónde se encuentra y su posible evolución hacia dónde va Es el primer paso antes de iniciar cualquier actividad diferente a los aspectos puramente operativos que permiten que la empresa funcione. Con el término gestión hacemos referencia aquí al conjunto de acciones didáctico organizativa necesaria para lograr una meta final determinada centrada en la construcción, transferencia y adquisición de conocimiento en la totalidad de las áreas de formación. El talento humano hay que entenderlo como la "capacidad para obtener resultados notables con el ejercicio de la inteligencia” (Moliner, M. 2004). En nuestro caso, dicha actividad es el adecuado desarrollo de las capacidades para la enseñanza (profesores) y el aprendizaje (estudiantes). Las acción gestora aquí estará fundamentada en una serie de decisiones en el ámbito de la antropología, lo disciplinar y la metodología didáctica.

Una herramienta muy útil para enfrentar estos nuevos retos es la llamada "gestión por competencias", la cual profundiza en el desarrollo y el involucramiento del talento humano, puesto que ayuda a elevar 
a un grado superior y de excelencia el potencial de cada uno de los colaboradores según su puesto de trabajo para lograr el objetivo global de alcanzar las metas de la organización.

La gestión por competencias es un enfoque que toma en cuenta los conocimientos, las habilidades, actitudes y comportamientos de tu capital humano ayudando a alinear sus objetivos y metas a los de la organización. Este tipo de administración contempla de forma integral la dimensión estratégica del negocio, la humana y la conductual, siendo así que las personas no solamente ocupan un puesto de trabajo y realizan actividades y tareas sino que los ayuda a desarrollarse para que puedan aportar lo mejor de sí a la empresa a través de competencias las cuales pueden ser un instrumento útil para la competitividad ya que son un factor de diferenciación en las empresas.

Es importante recordar que llevar a cabo esta práctica implica también una visión y colaboración de la dirección general para estructurar y guiar los equipos de trabajo, los procesos y las herramientas tecnológicas orientado estos recursos a la consecución de las metas empresariales globales.

Las competencias son un enfoque para la educación y no un modelo pedagógico, pues no pretenden ser una representación ideal de todo el proceso educativo, determinando cómo debe ser el proceso instructivo, el proceso desarrollador, la concepción curricular, la concepción didáctica y el tipo de estrategias didácticas a implementar.

En este sentido, (Tobón, 2007) como bien se expone, "el enfoque de competencias puede llevarse a cabo desde cualquiera de los modelos pedagógicos existentes, o también desde una integración de ellos”. Pag. 17.

"Es una característica de personalidad, devenidas de comportamientos, que generan un desempeño exitoso en un puesto trabajo."11 Todas las personas tienen un conjunto de atributos y conocimientos adquiridos o innatos que definen sus competencias para una cierta actividad. Sin embargo para descubrir las competencias no se necesita estudiar el perfil físico, psicológico o emocional, solamente interesan aquellas características que hagan eficaces a las personas dentro de la organización. (ALLES, Martha, 2008)

\section{MATERIALES Y MÉTODOS}

Los modelos de gestión basados en competencias son una herramienta valiosa para la administración de tu talento humano ya que te aporta dirección en procesos como:

a) Reclutamiento y Selección

b) Evaluaciones de desempeño del potencial

c) Planes de Desarrollo, Carrera y Sucesión

d) Compensaciones

Las competencias laborales permiten el desarrollo de los procesos dentro de la empresa al transformar la visión global (empresa-empleado) es decir, en lugar de trabajar cada uno para sus propios objetivos y tareas asignadas se cambia la perspectiva hacia una en donde el colaborador 
busca su crecimiento profesional y dentro de la empresa aumentando sus conocimientos, perfeccionando sus habilidades e involucrándose en conocer más a fondo a la organización, por lo que se convierte en un empleado más comprometido con el logro de los objetivos y metas tanto personales como organizacionales.

En el caso del reclutamiento y selección del personal, resulta muy útil contar con este enfoque ya que el reclutador se concentrará en aquellos postulantes que realmente cubran las competencias propias tanto del puesto como de la empresa tanto las básicas como las críticas. Es importante recalcar que aunque siempre van a existir áreas de oportunidades esta visión te ayuda a poder identificar el potencial de la persona y saber si una vez dentro de la empresa podrá fortalecer esas competencias.

De igual manera, la gestión por competencias es una guía esencial para los planes de carrera y sucesión en las empresas ya que como un resultado del desarrollo y seguimiento de tu equipo de trabajo podrás identificar a aquellos colaboradores que además de cumplir con los objetivos de su puesto han trabajado en fortalecer las competencias que son necesarias para acceder a un puesto de mayor jerarquía, en otra área y de igual manera saber en qué hay que seguir trabajando para alcanzar ese nivel.

Esto es importante ya que fomentarás el crecimiento de tu personal de manera interna, ocuparás vacantes con personal que ya colabora en la empresa y la conoce, y podrás ofrecer promociones de puesto y oportunidades a tus empleados cuando estén realmente preparados para ocupar ese cargo y su movimiento dentro de la empresa sea exitoso.

\section{RESULTADOS}

La gestión por competencias aporta un valor importante a la organización al maximizar el rendimiento de cada uno de los que conforman tu equipo de trabajo logrando generar en ellos el sentimiento de ser reconocidos por su esfuerzo y motivados en el cumplimiento de sus tareas. De igual manera podrás detectar qué áreas de oportunidad tiene cada persona en tu equipo y qué habilidades requiere desarrollar para avanzar a puestos de mayor responsabilidad en la empresa.

Los beneficios que traerá a tu compañía la implementación de este enfoque de gestión.

1) Facilitar la evaluación del desempeño al contar con una descripción más definida del puesto y las competencias que se requieren para el mismo.

2) Alinear las competencias específicas y generales que demanda la empresa con las que posee el colaborador.

3) Integrar los objetivos de desarrollo de la persona según su perfil de puesto con los de la organización consiguiendo un compromiso del empleado.

4) Fomentar un clima laboral de mutua cooperación y un modelo de liderazgo integrador y comunicativo. 
5) Incrementar la motivación y satisfacción del empleado al tener más claro cómo su trabajo y sus habilidades aportan valor a la empresa y colabora al cumplimiento de la misión.

6) Establecer un sistema de remuneración más justo, equitativo y eficiente, basado en su desempeño y desarrollo.

7) Desarrollar al capital humano como consecuencia de la gestión integral del capital humano desde la descripción de los puestos de trabajo, reclutamiento y selección, evaluación de desempeño hasta llegar al crecimiento profesional y plan de carrera.

8) Promover la formación progresiva y constante de tu capital humano.

9) Asegurar la congruencia entre los planes de entrenamiento y capacitación para los empleados y los requerimientos de la compañía.

10) Desarrollar equipos con las competencias necesarias para cada área operativa.

11) Identificar las fortalezas y áreas de oportunidad tanto a nivel organizacional como individual buscando siempre un ciclo de mejora.

12) Aumentar la productividad al optimizar resultados obteniendo ventajas competitivas.

13) Concientizar a los colaboradores de asumir la corresponsabilidad en el desarrollo de sus competencias profesionales.

En toda organización intervienen muchos elementos que deben estar bien coordinados para el logro de los objetivos de la misma, una organización es lo que las personas hacen de ella.

La comunicación es un conjunto de mensajes que se intercambian entre dos o más personas, siendo una el que transmite el mensaje y otra el que recibe el mensaje. La comunicación constituye una clave en las organizaciones representando un $75 \%$ de cada jornada laboral.

"La comunicación constituye la esencia del trabajo exitoso en equipo. La comunicación eficaz es el inicio del camino hacia el entendimiento, la interpretación y la acción. Por otro lado, la comunicación defectuosa puede conducir a la falta de comprensión mutua, los malos entendidos y eventualmente la inacción o acción inapropiada”. (CHANG, Richard Y., 1994)

La administración del talento humano ha ido evolucionando a medida que la globalización avanza, investigando y acumulando experiencias que están contribuyendo a la formación de un conjunto de conocimientos que ayudan a quienes dirigen al personal, a hacerlo más eficiente y efectivo.

La Administración del Talento Humano consiste en la planeación, organización, desarrollo y coordinación, así como también control de técnicas, capaces de promover el desempeño eficiente del personal, a la vez que debe incentivar a su talento humano no solo en el ámbito profesional y económico, sino también en las áreas sociales, culturales, turísticas y deportivas, ya que son parte de

"La capacidad de la persona que entiende y comprende de manera inteligente la forma de resolver problemas en determinada ocupación, asumiendo sus competencias (habilidades, conocimientos y actitudes) destrezas, experiencias y aptitudes, estas capacidades se adquieren con el entrenamiento, la educación y la experiencia de cada uno y que 
lo hacen potencialmente productivo. Esto es la inversión que la empresa realiza en el entrenamiento del personal como medios para lograr el objetivo general de incrementar la productividad" (http:// www.ced.ucn.cl/s_taller_2005/temas/talento_humano.htm, s.f.)

A medida que se acerca el nuevo milenio, las organizaciones se desarrollan cada vez más, con una clara tendencia hacia la unificación de principios y de formas de hacer negocios. Los procesos productivos, las acciones comerciales, la planificación financiera y otros factores son similares en las diferentes empresas, pertenezcan o no al mismo sector o al mismo país. En la actualidad, y por este motivo, es posible que una organización implante en otra un sistema de gestión que esté funcionando con éxito, realizando simplemente algunas modificaciones de adaptación.

Cada empresa desarrolla sus actividades en un entorno propio, pero las técnicas de gestión de los negocios han alcanzado un nivel de unificación global que no se había conocido antes. Por ello, es fundamental que el equipo directivo sea consciente de su ubicación para poder conocer y definir el entorno de la empresa dónde se encuentra y su posible evolución hacia dónde va. Es el primer paso antes de iniciar cualquier actividad diferente a los aspectos puramente operativos que permiten que la empresa funcione.

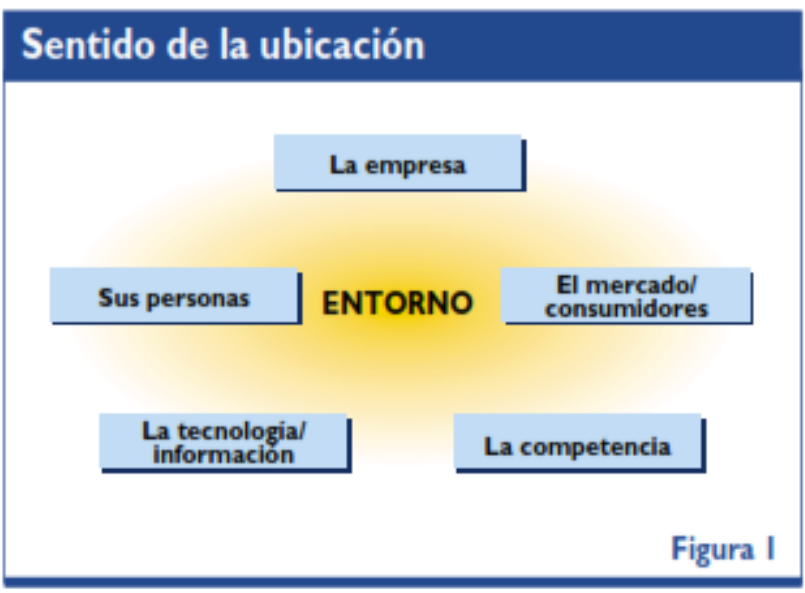

\section{ILUSTRACIÓN 1 \\ SENTIDO DE LA UBICACIÓN \\ Los autores}

Hoy, la fuerza de las organizaciones proviene de sus personas y el reto es, precisamente, formar e integrar un buen equipo de trabajo.

Las organizaciones no son un ente abstracto que produce bienes o servicios; y en algunas ocasiones hasta beneficios, afortunadamente. Las empresas son un conjunto de personas que llevan a cabo actividades para la consecución de objetivos planificados. En un barco, la flotación es posible gracias al sustento de la estructura sumergida y no por lo que se ve desde fuera; también la fuerza que sostiene una organización se encuentra en las personas, en sus sistemas de integración, en sus capacidades y en su grado de compromiso personal y hacia la organización. En muchas ocasiones, el éxito o fracaso no reside en lo que se percibe a simple vista en las organizaciones, sino en el conocimiento y aprovechamiento de 
las capacidades y habilidades de las personas para la consecución de los objetivos que la empresa haya fijado.

\section{DISCUSIÓN}

Implementar en la empresa este enfoque de gestión del talento humano por competencias se vuelve una tarea más sencilla y eficiente cuando se administra mediante una solución tecnológica como SuccessFactors que integra los diversos procesos referentes a tu capital humano ya que esto te permitirá tener una visión integral logrando el objetivo final que es contar con un equipo de trabajo desarrollado, preparado y potencializado, el cual estará motivado y comprometido a formar una sinergia con la empresa para alcanzar las metas y objetivos.

A través de una política de recursos humanos se busca la formación de personas que vayan creciendo profesional y personalmente dentro de la organización. Siguiendo el enfoque de competencias, el plan de carreras es un método que determina las tareas organizativas y los conocimientos y habilidades clave a desarrollar para un desempeño superior, ya sea en el puesto de trabajo actual o en otro de la organización. Con el canal de información que proporcionan las competencias es posible identificar las características y aptitudes de cada persona y de cada puesto para proponer un plan de carrera adaptado a ellas.

Es fundamental entonces transformar nuestra visión, desde la economía a la economía humana, desde el Capital de Trabajo hacia el desarrollo, potencialización del Capital Humano, que es lo que en esta década le dará la única ventaja competitiva sustentable en el tiempo a la empresa.

Por otro lado, aunque el Sistema de Certificación de Competencias sea difícil de desarrollar y de llevar a la realidad, debe ser un esfuerzo continuo y sobre todo compartido por los sectores gubernamentales, privado y educativo, de tal manera que una alianza entre ellos derive en el incremento de la productividad, las ganancias, el empleo y el sistema de capitales basados en conocimientos que se buscan hoy en día.

\section{Referencias}

1. ALLES, M. (2008). Dirección Estratégica de Recursos Humanos, Gestión por competencias. Argentina: Ediciones Gránica.

2. CHANG, R.Y. (1994). Trabajar en equipo para triunfar. Argentina: Ediciones Granica S.A.

3. Universidad Católica del Norte (2005). Talento Humano. Chile:CEDUCN. Recuperado de http://www.ced.ucn.cl/s_taller_2005/temas/ talento_humano.htm.

4. Moliner, M. (2004). Diccionario del uso del español. Tomos A-He I-Z. Madrid: Editorial Gredos.

5. Tobón, S. (2007). El enfoque complejo de las competencias y el diseño curricular. Acción pedagógica, 16(1), 14-28. 